\title{
Possibilities in State Event Modelling of Hybrid Systems
}

\author{
Andreas Körner*, Stefanie Winkler, Felix Breitenecker \\ Institute of Analysis and Scientific Computing, TU Wien, Wiedner Hauptstraße 8-10, \\ 1040 Vienna, Austria; *andreas.koerner@tuwien.ac.at
}

SNE 28(3), 2018, 109-111, DOI: 10.11128/sne.28.sn.10429 Received: Sept. 15, 2016 (Selected EUROSIM Congress 2016 Postconf. Publ.), Revised Sept. 15, Accepted: Sept. 20, 2018 SNE - Simulation Notes Europe, ARGESIM Publisher Vienna, ISSN Print 2305-9974, Online 2306-0271, www.sne-journal.org

Abstract. This work-in-progress paper illustrates the idea of the conceptional separation between a mathematical model and a simulation model for state event models of dynamic hybrid systems. The traditional modelling and simulation process starts with a mathematical model, followed by an implementation of the model in a certain simulation environment and ends with the parametrisation of the numerical attributes followed by the simulation run. Current trends in simulation environments tends to go in the direction of graphical oriented modelling descriptions neglecting needs of conceptional (mathematical) models at the beginning of the process. This limits the modelling process and restricts efficiency. For comparison or benchmarking simulation environments as well as modelling languages, approaches and optimization for hybrid models, a conceptional model offers a framework to review these aspects.

\section{Introduction}

Modelling and simulation can not be used separately. Especially in the field of hybrid models this aspect is even more important, because in many cases the description refers to both the modelling and the simulation. Often algorithms are used to specify hybrid descriptions. There are various areas applying hybrid system models to analysis system behaviour, e.g. science, engineering, robotic systems, traffic management systems, sequential operations as well as embedded systems. [1]

\section{Hybrid Modelling}

The term hybrid has different meanings, the model structure or the mixture of used environments. In this paper the term hybrid means variation in the structure of the model. There are several changes in the model framework possible, which is discussed in details in [2]. The offered framework in this work discusses and analyzes the possibilities of hybrid modelling.

\section{State Event Modelling}

This modelling approach is closely related to hybrid modelling and provides a particular mathematical description regarding the numerical handling and execution of hybrid phenomena in a model description. The basic approach starts with the assumption of a certain dynamical system and performs the change in the model structure by a particular event depending on the state of the system. This state event changes the model framework and performs the continuing simulation run with changed model framework.

Hybrid modelling and state event modelling, address the same branch of systems but provide different approaches for modelling and simulation. Both modelling methods describe time sequential processing of different model descriptions. State event modelling, normally restricts the range of model description to ordinary differential equations (ODEs) or algebraic differential equations (DAEs), whereas the hybrid modelling approach can include different model descriptions and methodologies. In the following section the architecture of hybrid models and state event models respectively will be discussed. 


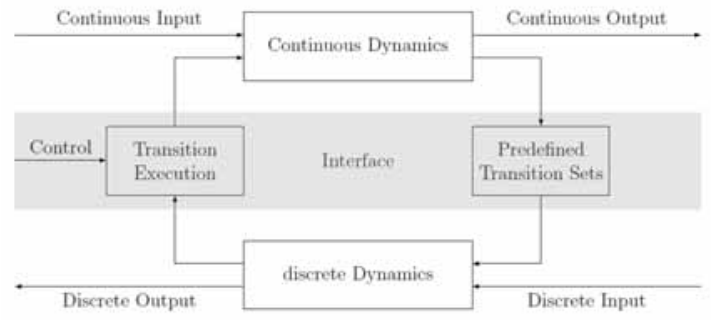

Figure 1: System architechture of Hybrid Dynamical Model.

\section{Hybrid Model Architecture}

Continuous controlled hybrid systems cover most of the discussed hybrid phenomena. The autonomous switches and jumps are covered in this environment, as required in [3], as well as the structure-variant systems introduced in [4]. Therefore the provided mathematical environment covers several system behaviours except for external controlled behaviour. An illustration of the architecture of this model description is given in Figure 1.

This system architecture requires a particular mathematical environment, which offers illustrated functionality. As a mathematical environment, the hybrid system consists of a given set of differential algebraic equations, which are assigned to the current state in which the system is currently working. The mathematical set-up is given by the following Definition.

The sixtupel $\left(L, X, W, F_{L}, G, J\right)$ denotes the generalised dynamic hybrid system automaton. $L$ denotes the finite set of states, $X$ the collection of corresponding state spaces, $W$ the set of collections of communication spaces and $F_{L}$ the set of differential algebraic equations. The collections $X$ and $W$ implements the opportunity to have in each state $l \in L$ a particular state space and vector of communication variables. Furthermore $\mathscr{F}$ as the set of differential and algebraic equations with the elements $(f, g) \in \mathscr{F}$. This tuples represents functions $f: \mathbb{R}^{n} \times \mathbb{R}^{m} \times \mathbb{R}^{\ell} \times \mathbb{R}^{r} \times \mathbb{R}$ the right side of the differential equation

$$
\dot{x}=f(x, u, z, p, t)
$$

and $g: \mathbb{R}^{n} \times \mathbb{R}^{m} \times \mathbb{R}^{\ell} \times \mathbb{R}^{r} \times \mathbb{R} \rightarrow \mathbb{R}^{u}$ represents the algebraic equation

$$
g(x, u, z, p, t)=0
$$

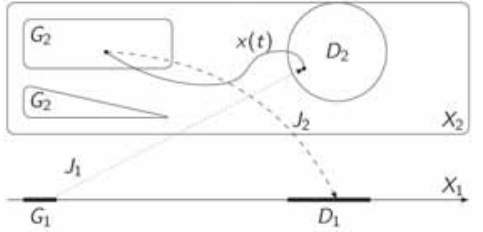

Figure 2: Example of a State Space Transition in an low dimensional Situation.

This set of differential algebraic equations represents the continuous dynamic of the model in a particular state. The discrete dynamic represents the transition from one continuous description to the other, either autonomous or controlled.

The collection of sets $G$ and $J$ implements the connection between the continuous state and the discrete transitions which are related by guard maps $G_{l}$ and jump transition maps $J_{l}$. The transitions are enabled by entering the guard region and the jump is performing the transition from one state space in the location $l$ to the other state space in the location $l^{\prime}$. A simple illustration for the one and two dimensional case is presented in Figure 2.

The introduced mathematical framework allows a model description on an abstract layer without limitation of simulation environments but with procedural structured situation. Therefore this mode framework is called a structural model.

\section{Prospects of a Mathematical Formalised Simulation Model}

Formalizing a mathematical model description offers possibility for theoretical consideration independent of a particular simulation environment. For a state event approach of a hybrid system this offers the possibility of separating numerical and modelling tasks in two different layers:

- Model Framwork

- Numerical Framwork

On the one hand this separation allows a better understanding of the abilities of a simulation environment, especially for benchmarking this separation is helpful. On the other hand the conceptual structure enables a range of theoretical considerations of models and scenarios. The particular type of transition in a hybrid model can be observed but also considerations regarding 


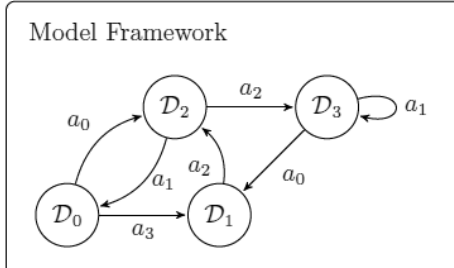

Figure 3: Illustration of the Structural Model Description.

optimization and improvement of the model itself can be addressed.

A hybrid model structured as illustrated in Figure 3 can not be optimized by optimizing each state for itself. The overall structure has to be optimised and this in a way, that maybe transition has to be redefined or particular continuous dynamics in an arbitrary state has to be reformulated. This way is enabled having a mathematical model description available.

\section{Conclusion and Outlook}

This work-in-progress paper shows the beginning of a conceptual work to formalise simulation models for state event modelling of hybrid systems. This contribution shows the first steps towards a conceptual simulation environment. A more detailed consideration related to mathematical characterizations of state events is done in [2].

The characterization of hybrid state event modelling should also support benchmarking and categorization of different existing optimization approaches as discussed in[1] and [5]. The Framework enables to formulate a general concept regarding optimization. The actions defined above lead to different algorithms as well as different cost functions. The questions is how to optimize a hybrid model in detail, perhaps using a weighted cost function including all subsystems. Is the optimization of every single subsystem the optimization of the complete hybrid system?

\section{References}

[1] Barton PI, Lee CK. Modeling, Simulation, Sensitivity Analysis, and Optimization of Hybrid Systems. ACM Transactions on Modeling and Computer Simulation. 2002;12:256-289.

[2] Körner A. Mathematical characterisation of state events in hybrid modelling. Ph.D. thesis, Technische Universität Wien. 2015.

[3] Ding XC. Real-time optimal Control of Autonomous Switched Systems. Ph.D. thesis, Georgia Institute of Technology. 2009.

[4] El-Ghezawi O, Zinober A. Variable Structure Systems and System Zeros. 1981. Research Report No. 157, University of Sheffield, England.

[5] Barton PI, Lee CK, Yunt M. Optimization of hybrid systems. Computer and Chemical Engineering. 2006; 30:1576-1589. 